This item was submitted to Loughborough's Research Repository by the author.

Items in Figshare are protected by copyright, with all rights reserved, unless otherwise indicated.

\title{
Accurate characterisation of hole size and location by projected fringe profilometry
}

PLEASE CITE THE PUBLISHED VERSION

https://doi.org/10.1088/1361-6501/aab67e

PUBLISHER

(C) IOP Publishing

VERSION

AM (Accepted Manuscript)

LICENCE

CC BY-NC-ND 4.0

\section{REPOSITORY RECORD}

Wu, Yuxiang, Harshana Dantanarayana, Huimin Yue, and Jonathan Huntley. 2018. "Accurate Characterisation of Hole Size and Location by Projected Fringe Profilometry". Loughborough University. https://hdl.handle.net/2134/32270. 


\section{Accurate characterisation of hole size and location by}

\section{projected fringe profilometry}

Yuxiang $\mathrm{Wu}^{\mathrm{a}}$, Harshana G. Dantanarayana ${ }^{\mathrm{b}}$, Huimin Yue ${ }^{\mathrm{c}}$, Jonathan M. Huntley,

${ }^{a}$ Xidian University, School of Physics and Optoelectronic Engineering, Xi’an, Shaanxi, 710071, China;

${ }^{\mathrm{b}}$ Wolfson School of Mechanical, Electrical and Manufacturing Engineering, Loughborough University, Loughborough, Leicestershire, LE11 3TU, UK;

${ }^{\mathrm{C}}$ University of Electronic Science and Technology of China, Chengdu City, Sichuan Province, China.

\section{ABSTRACT}

The ability to accurately estimate the location and geometry of holes is often required in the field of quality control and automated assembly. Projected fringe profilometry is a potentially attractive technique on account of being non-contacting, of lower cost, and orders of magnitude faster than the traditional coordinate measuring machine (CMM). However, we demonstrate in this paper that fringe projection is susceptible to significant (hundreds of $\mu \mathrm{m}$ ) measurement artefacts in the neighbourhood of hole edges, which give rise to errors of a similar magnitude in the estimated hole geometry. A mechanism for the phenomenon is identified based on the finite size of the imaging system's point spread function and the resulting bias produced near to sample discontinuities in geometry and reflectivity. A mathematical model is proposed, from which a post-processing compensation algorithm is developed to suppress such errors around the holes. The algorithm includes a robust and accurate sub-pixel edge detection method based on a Fourier descriptor of the hole contour. 
The proposed algorithm was found to reduce significantly the measurement artefacts near the hole edges. As a result, the errors in estimated hole radius were reduced by up to one order of magnitude, to a few tens of $\mu \mathrm{m}$ for hole radii in the range 2-15 mm, compared to those from the uncompensated measurements.

Keywords: projected fringes, profilometry, hole measurement, edge detection, Fourier descriptor

* Corresponding author, email: j.m.huntley@lboro.ac.uk. 


\section{Introduction}

Accurate estimation of size and location of holes [1,2] on different kinds of surfaces is often required in industrial quality control and automated assembly, since a small deviation of diameter and position estimation may lead to a mismatch of assembly components. This process is usually performed by a mechanical coordinate measuring machine (CMM). Mechanical CMMs measure object shapes by physically probing points at different locations on objects [3]. Although it is capable of producing the highly accurate measurements that industry demands, its major shortcoming includes a relatively long measurement time and the need to contact the test surface.

Projected fringe profilometry [4-6] has the advantages of being non-contact, fast and costefficient. 3D scanners based on fringe projection can measure millions of points on the surfaces of objects with dimensions ranging from a few $\mathrm{cm}$ to a few $\mathrm{m}$, in just a few seconds, with an accuracy of typically a few tens of $\mu \mathrm{m}$. Fringe projection therefore has the potential to be an effective alternative approach to estimate the sizes and locations of holes. However, it remains to be proven how accurate and robust such estimations are when compared to those obtained from the industry standard mechanical CMMs. The presence of discontinuities in reflectivity and specimen geometry has previously been linked to systematic errors in measurements from fringe projection systems [7-9]. The effect on the accuracy of hole characterisation has not, however, been reported previously, apart from a recent conference paper by the same authors [9] on which the current paper is based.

In this paper, results from an experiment to compare fringe projection measurements with those from a mechanical CMM are presented. These demonstrate that very significant errors (around $0.3 \mathrm{~mm}$ radii mean error for $8 \mathrm{~mm}$ radius holes) can in fact arise in such an application of projected fringe profilometry. The experiments were done on an in-house 
fabricated test panel with a range of holes of varying sizes, following a procedure described in section 2. In order to estimate the radii and the positions of the holes automatically, a standard image processing technique was employed to process the coordinate data. In section 3, we provide a possible explanation for the underlying cause of the errors and propose a correction algorithm. A fundamental requirement of this correction algorithm is a robust subpixel edge detection method, so in section 4, a robust contour estimation method based on a Fourier descriptor approach is proposed. In section 5 we present results from applying the algorithms outlined in sections 3 and 4 to the test data and demonstrate dramatic (up to $11 \times$ ) reduction in errors in the estimated radius values.

\section{Experimental}

An aluminium test panel of dimensions $300 \times 300 \times 10 \mathrm{~mm}^{3}$ was manufactured with a set of through holes, with diameters ranging from $30 \mathrm{~mm}$ down to $1 \mathrm{~mm}$, as shown in Figure 1. Apart from the holes with the two smallest diameters (1 $\mathrm{mm}$ and $2 \mathrm{~mm}$ ), which were measured by means of an optical CMM (SmartScope FLASH 200), the radius and the centre position of each hole on the panel was estimated using a mechanical CMM (LK Ultra Metris). As the hole radius increased from $2 \mathrm{~mm}$ to $15 \mathrm{~mm}$, the number of CMM probe touch points around the hole edges was increased from 46 points to 909 points. The CMM tip had a diameter of $0.5 \mathrm{~mm}$ and the probe touched points $1 \mathrm{~mm}$ below the surface (inside the hole) during the measurement. The CMM recorded the $X, Y$ and $Z$ position of each touch point around the circumference, and estimated the hole radius and centre position after fitting a circle to the measured points. The measurement process, facilitated by the prior knowledge of the hole positions, was mainly manual and the whole process took more than 2 hours to complete. 
A Phase Vision Quartz 1200 fringe projection 3D scanner was then employed to obtain a dataset of $X, Y$ and $Z$ coordinates covering the surface of the test panel. Following calibration, the scanner projected sinusoidal fringe patterns on to the test object with a reverse exponential fringe sequence, and a reverse exponential least-squares temporal phase unwrapping (TPU) algorithm [10,11] was used to extract the unwrapped phase from the recorded fringe patterns. The measurement system has a working distance of 1.8-2.8 $\mathrm{m}$, and produces point clouds of $2048 \times 2048$ pixels with a $100-\mu$ m measurement accuracy.

Prior to measurement, a thin layer of white developer powder was applied to the upper face of the plate to produce a more diffusely scattering surface with reduced specular 'hot spots'. Care was taken to avoid the powder from entering into the holes themselves. The holes were detected automatically by applying image processing techniques to the 'texture image', i.e. the intensity distribution produced by uniform lighting of the scene, which is also recorded as part of the scan. The standard image processing procedures that were used included the following four steps, which are also illustrated in Figure 2:

1. Detect initial hole edges with a Canny operator [12],

2. Label each disconnected edge,

3. Fit ellipses to the clusters of connected edge points to detect whether it is a hole,

4. Determine the centre and identify the pixels on the circumference of each detected circular hole.

After these image processing steps on the texture image, the radius of a given hole was estimated by least squares fitting of the equation for an ellipse to the $X, Y$ and $Z$ coordinate data on the pixels identified as lying on the hole's perimeter. Figure 3 shows the estimated results thus obtained over a small portion of the test object, in this case for the set of holes 
with a nominal $8 \mathrm{~mm}$ radius. The estimated radius is always significantly higher than the true radius (where the true radius is taken to be that determined by the CMM measurements), with a mean error of approximately $0.3 \mathrm{~mm}$. This is significantly higher than the intrinsic measurement accuracy of the scanner (ca. $100 \mu \mathrm{m}$ ) and is also 12 times larger than the measured mean hole circularity of approximately $0.025 \mathrm{~mm}$.

\section{Discontinuity-induced measurement artefact (DMA) and the compensation model}

A clue to the underlying cause of the overestimated radius can be found in the distribution of measured height in the vicinity of a hole. Figure 4(a) shows this distribution for a hole of radius $4 \mathrm{~mm}$, after subtraction of a best-fit plane. A systematic measurement error is visible which has a peak value of over $0.5 \mathrm{~mm}$ in both the positive and negative directions, close to the edge of the hole, and which is many times the intrinsic random error of the scanner. One side of the hole edge appears to be lowered while the other side is raised giving rise to an apparent approximately elliptical shape to the hole edge. The minor axis of the ellipse may be close to the true radius $R_{0}$, as illustrated in Figure 4(b), but the major axis will be larger. Therefore, in the presence of this systematic error, the estimated radius $R$ produced by fitting a circle to the edge coordinates will be consistently larger than $R_{0}$.

The cause of the systematic error shown in Fig. 4(a) is believed to be the imperfect averaging produced when significant intensity variations occur over a length scale comparable to the diameter of the camera's point spread function (PSF). This effect has recently been analysed for the case of discontinuities in reflectivity on otherwise continuous surfaces in [8]. The case of a hole is in general more complex because in addition to the discontinuity in reflectivity there are geometrical discontinuities (in both depth and in slope). However, a 'black hole', i.e. one for which no light is back-scattered from within the hole, will be indistinguishable from a perfectly absorbing black circle on a continuous uniformly scattering surface. It therefore 
seems plausible that the method developed in [8] is also applicable to the correction of measurement errors around holes, at least for cases where the scattered light intensity from within the hole is much less than from the material immediately surrounding it.

A simple 1-D example to illustrate the effect is shown schematically in Figure 5. Figure 5(a) shows a cross section through an image of a uniformly-illuminated plate with a hole. The vertical axis is normalised intensity, denoted here $I(x)$, and the horizontal axis represents position, $x$, along the plate. In this example, the hole occupies the region $-D / 2<x<D / 2$, where $D=0.4$. The plate is then illuminated with a sequence of fringe patterns, one of which is shown in Fig. 5(b). If for example the reversed exponential sequence [11] is used to provide an independent unwrapped phase value at each camera pixel, a sequence with 8,7,6,4 fringes across the field could be projected, each of which would have four phase shifts. Figure 5(b) then represents the first of these 16 patterns, consisting of eight fringes and zero phase shift.

The unwrapped phase profile computed from these patterns, $\omega_{x}(x)$, is shown in Fig. 5(c). The continuous line represents $\omega_{x}(x)$ normalised onto the range $-\pi$ to $+\pi$; the dashed-dotted line is the phase that would have been measured if the plate had been continuous, but which is not calculable due to the absence of measurable intensity from the hole region. The subscript ' $x$ ' in $\omega_{x}$ refers to the phase resulting from fringes oriented normal to the $x$ axis. In the full 2-D case considered later, $\omega_{y}$ will be used to refer to the unwrapped phase map from fringes projected normal to the $y$ axis.

We have assumed thus far that the camera recording the images has a PSF of negligible diameter compared to the field of view. Consider now the situation where this is not the case, for example due to significant defocus of the camera. Point P in Fig. 5(c) is a point on the sample well away from the hole, with true unwrapped phase $\omega_{x}^{\left({ }^{(P)}\right.}$. The horizontal bar 
represents the spatial extent of the PSF. The signal at P now contains contributions from the range of $\omega_{x}$ values falling within the range of the bar, however since the sample is uniformly reflective over the PSF region, as seen in Fig. 5(a), the contribution from the regions to the left and right of $\mathrm{P}$, where $\omega_{x}$ is respectively greater than and less than $\omega_{x}{ }^{(\mathrm{P})}$, can be expected to largely cancel out. Point $\mathrm{Q}$ also receives signal from the region of the sample falling within the range of the bar centred on Q. However, as Q lies on the left hand edge of the hole, signal only comes from scattering points lying to the left of Q. The contributions to the detected signal all have $\omega_{x} \leq \omega_{x}^{(Q)}$ and the measured phase is thus biased in the downward direction. The opposite happens at point $\mathrm{R}$ where there is a positive bias in the measured phase.

A model for the measured phase from this finite sized PSF case is shown in Fig. 5(d). Here we have assumed that the measured phase is a convolution of the intensity-weighted true phase, $g(x)$, with the camera's PSF $h(x) . g$ is thus the product of the true phase (Fig. 5(c)), and the intensity signal (Fig. 5(a)), and $h$ was chosen to be a rectangular ('top hat') function of width 0.2 .

The measured profile across the hole which is obtained by removal of the linear ramp and appropriate scaling, then has positive and negative artefacts next to the hole which are reminiscent of those shown in Fig. 4(a). The example in Fig. 5(e) is the profile that would be obtained for the case of a $45^{\circ}$ projection angle, and the artefacts are seen to be a significant fraction of the field of view.

It should be noted that this effect is not limited to any particular scanner or phase shifting/unwrapping algorithm but is an optical phenomenon related to the finite size of the imaging system's point spread function. Factors influencing the PSF diameter include lens aberrations, the finite pixel size of the image sensor, and ultimately diffraction. In practice, defocus is likely to be the biggest contributor to PSF size for scattering regions within the 
measurement volume that lie a significant distance from the plane of best focus. The effect is thus likely to be present in any triangulation based optical sensor, including light stripe sensors.

\subsection{The compensation model}

It is interesting to test the validity of the error source proposed above, and ultimately to derive a compensation algorithm to remove the errors. In this section we consider how this might be attempted by means of a simple mathematical model.

Figure 6 is a schematic of the top view of a plate containing a hole. The 1-D example in Fig. 5 can be regarded as a cross-section through the centre of this hole, along the line $y=0$, where the origin for the $(x, y)$ coordinate system lies at the centre of the hole. If the PSF has radius $R_{P}$, then only those image plane points within a distance $R_{P}$ of the edge of the hole are affected by the presence of the hole. This region is denoted the 'PSF zone' in Fig. 6.

As in [8] it will be assumed that the phase measured by the system at an arbitrary point in the PSF zone with coordinates $\left(x_{c}, y_{c}\right)$ may be modelled as a weighted average of the true phase values lying within the PSF footprint, shown as the grey shaded circular region in Fig. 6 . The weighting function is chosen to be the product of $I$ and the PSF, as this is proportional to the signal level (number of photons, and hence number of photoelectrons in the image sensor) that will be detected at point $\left(x_{c}, y_{c}\right)$ from point $(x, y)$ for each of the recorded fringe patterns. The output of the model for the phase $\bar{\omega}_{j}(j=x, y)$ recovered at point $x=x_{c}, y=y_{c}$, may thus be written as the convolution integral

$$
\bar{\omega}_{j}\left(x_{c}, y_{c}\right)=\frac{\iint_{-\infty}^{\infty} \omega_{j}(x, y) \cdot I(x, y) \cdot h\left(x-x_{c}, y-y_{c}\right) \mathrm{d} x \mathrm{~d} y}{\iint_{-\infty}^{\infty} I(x, y) \cdot h\left(x-x_{c}, y-y_{c}\right) \mathrm{d} x \mathrm{~d} y}
$$


where $\omega_{j}$ and $I$ are respectively the unwrapped phase and texture images that would be measured by an idealised camera with no image blur. $h$ is the PSF, and the denominator represents a normalisation factor. For many optical systems, the PSF may be modelled as a Gaussian [13]:

$$
h(x, y)=a \mathrm{e}^{-\frac{x^{2}}{2 c^{2}}} \mathrm{e}^{-\frac{y^{2}}{2 c^{2}}}
$$

where the parameter $a$ is another normalisation factor, and $c$ controls the width which is assumed to be identical along both the $x$ and $y$ axes. If we define the radius $R_{P}$ as being the half width at one tenth of maximum (HWTM), then $R_{P}=\sqrt{2 \ln 10} c \approx 2.146 c$.

In order to test the validity of this model, and to develop an error compensation method, estimators $\widehat{\omega}_{j}$ and $\hat{I}$ are required for the $\omega_{j}$ and $I$ functions inside the integral of equation (1). In the current paper this is done by least squares fitting polynomial surfaces to the measured phase and texture images in the region immediately outside the PSF zone, i.e. the closest region where the data are unaffected by the hole edge. This region is denoted the 'Least Squares Fitting' (LSF) zone in Fig. 6. The best fit polynomial coefficients from the LSF zone are then use to estimate $\omega_{j}$ and $I$ in the PSF zone [8].

In general, PSF zones lie on both sides of a discontinuity. Figure 6 only shows one side of the PSF zone, however, because the other side is inside the hole and will not contain any valid data. The measured phase data within the hole is therefore simply masked out. The unwrapped phase measured by the shape measurement system will be denoted $\widetilde{\omega}_{j}$. Within the PSF zone, $\widetilde{\omega}_{j}$ is affected by DMA error, so the unwrapped phase at all pixels within the PSF zone needs to be modified. 
As with the PSF zones, LSF zones lie on either side of the PSF zone, but only the solid material side is considered in the case of a hole. The width of the LSF zone is also chosen to be $R_{P}$ so that it contains sufficient information for reliable fitting whilst also keeping the information local enough. The data in the LSF zone is used to compensate for the phase errors in the pixels within the PSF zone using the model introduced below. This is based on a recently-proposed model to compensate for the DMA errors in the neighbourhood of discontinuities in reflectivity [8].

Once the extrapolated unwrapped phase $\widehat{\omega}_{\mathrm{j}}$ is calculated from the LSF zones and the

modelled phase $\bar{\omega}_{j}\left(x_{c}, y_{c}\right)$ is obtained by integration using equation (1), the error $\varepsilon_{\widetilde{\omega}_{j}}$ that will be present in the measured $\widetilde{\omega}_{j}$ at pixel $\left(x_{c}, y_{c}\right)$, due to the neighbouring hole, is estimated as follows:

$$
\varepsilon_{\widetilde{\omega}_{j}}=\bar{\omega}_{j}-\widehat{\omega}_{j}
$$

The measured $\widetilde{\omega}_{j}$ value is corrected as

$$
\widetilde{\omega}_{j}{ }^{\prime}=\widetilde{\omega}_{j}-\varepsilon_{\widetilde{\omega}_{j}}
$$

A corrected position vector for the scattering point is then computed from the $\left(\widetilde{\omega}_{x}{ }^{\prime}, \widetilde{\omega}_{y}{ }^{\prime}\right)$ coordinates, using the model for the scanner that provided the original position vector.

\subsection{DMA error compensation algorithm for holes}

The proposed algorithm to compensate for the DMA error on and around a hole relies on detection of discontinuous transitions in intensity from the texture image, followed by estimation of the resultant artefact from phase and intensity values in the LSF zones, rather than by attempting to identify the artefacts directly from the measured point cloud. The steps are summarized below. 
a. Calculate an initial estimate of the radius and the centre coordinates for each hole in image plane coordinates (i.e., with units of pixels) from the texture image, using the procedure described in section 2. For each hole, choose a sub-image window containing only the hole and sufficient surrounding material (the need for this is explained later) according to the initial radius and the centre pixel estimates of the hole. In this study, to ensure consistency across all the datasets, the sub-image window was chosen to be a square with sides 3 times the diameter of the hole centred on the hole centre. It should be pointed out, however, that much smaller sub-image window dimensions also work well in practice.

b. For each sub-image (i.e., for each hole), detect the hole edge using an edge detection method with sub-pixel precision. This method should provide the hole edge as pixel points and the hole centre to sub-pixel accuracy, in addition to the local tangential direction at each sub-pixel edge point.

c. For each sub-image, dilate the Canny edge image in order to obtain the mask of the PSF region. The dilation width should be consistent with the $R_{P}$ value of the PSF of the camera (see section 3.1). For the results presented here, $R_{P}$ was obtained by measuring the edge response function of the camera, and subsequently fitting a Gaussian curve to the first order difference of the intensity image [13].

d. For each sub-image, model the DMA error at pixels within the PSF zone as follows. For each pixel within the PSF zone:

(1) Check whether the chosen pixel is inside or outside the hole edge. The check is conducted by comparing $d_{1}$ (the distance between the hole centre and the pixel location) and $d_{2}$ (the distance between the hole centre and the sub-pixel edge point closest to the chosen pixel). If $d_{1}<d_{2}$, the chosen pixel is inside the edge and the phase is masked out as an invalid data point. 
(2) If the chosen pixel is outside the edge $\left(d_{1}>d_{2}\right)$, select its nearest sub-pixel edge point. This is illustrated in Fig. 7(a), where the nearest sub-pixel edge point is point 2.

(3) Create a mask to choose the data in the LSF zone (Zone 1 in Fig. 6) from which $\widehat{\omega}_{\mathrm{x}}, \widehat{\omega}_{\mathrm{y}}$ and $\hat{I}(x, y)$ will be calculated by extrapolation within the PSF zone. Zone 1 is defined by the constraints $r_{e}(\phi)+R_{P}<r<r_{e}(\phi)+2 R_{P}$ and $\phi_{c}-\alpha<\phi<\phi_{c}+\alpha$, where $r_{e}(\phi)$ is the radius function of the sub-pixel edge points. $(r, \phi)$ and $\left(r_{c}, \phi_{c}\right)$ represent the polar coordinates of an arbitrary pixel and of point $\left(x_{c}, y_{c}\right)$, respectively, with respect to the hole centre. $\alpha$ is an angular parameter to define the boundaries of Zone 1. In this study, the outside arc length of Zone 1 was set to be $2 R_{P}$, hence $\alpha\left(\phi_{c}\right)=2 R_{P} /\left[r_{e}\left(\phi_{c}\right)+2 R_{P}\right]$.

(4) Rotate and translate the image plane, and the mask plane from the previous step, to a coordinate system $\left(x^{\prime}, y^{\prime}\right)$ with its origin at the sub-pixel edge point closest to the chosen pixel, and its $x^{\prime}$-axis normal to the tangent at the sub-pixel edge point and pointing outwards from the hole as shown in Fig. 7(b).

(5) Calculate the modelled omega $\bar{\omega}_{x}$ at the current pixel $\left(x_{c}, y_{c}\right)$ using the integration model in Section 3.1, and hence obtain corrected unwrapped phase values $\widetilde{\omega}_{x}{ }^{\prime}$ and $\widetilde{\omega}_{y}{ }^{\prime}$ using equations (3) and (4).

e. Convert the corrected phase values to corrected coordinates $\left(X^{\prime}, Y^{\prime}, Z^{\prime}\right)$ at all pixels within the PSF zone using the original scanner calibration model.

To estimate the radius and the centre location, a 3D circle fitting is employed on the compensated $\left(X^{\prime}, Y^{\prime}, Z^{\prime}\right)$ data at the sub-pixel edge points. 


\section{Fourier descriptor contour estimation method}

In order for the above DMA error compensation method to work effectively, it is essential to employ a robust sub-pixel edge detection algorithm on the texture sub-images. One previously-published method [14] was used initially that worked with some success for larger holes, but which had clear defects when applied to smaller holes (2 mm or smaller radius). An example is shown in Figure 8 (green dots) for three different hole radii: although the detected edge location appears reasonable to the eye for the $4 \mathrm{~mm}$ hole (Fig. 8(a)), at $2 \mathrm{~mm}$ radius (b) the shape of the hole edge appears to be no longer circular, and for both this and the $1 \mathrm{~mm}$ sub-image (Fig. 8(c)) the detected edge appears qualitatively to lie within the true edge, which would lead to underestimation of the hole size. For this reason an alternative 'Fourier Descriptor' approach has been investigated based on expansion of the hole radius $r_{e}$ as a Fourier series in $\phi$. The theoretical background to this approach, and results of simulations to validate the method, are described in the next two sub-sections.

\subsection{Mathematical model of the Fourier Descriptor method}

The method described here models the hole edge in a texture image $I(x, y)$ as a closed contour of arbitrary shape, with assumed uniform intensity magnitudes of $I_{1}$ and $I_{2}$ inside and outside the contour, respectively (see Fig. 9). The contour can be specified as a Fourier series as follows:

$$
r_{e}(\phi)=a_{0}+\sum_{k=1}^{N_{f}}\left[a_{k} \cos (k \phi)+b_{k} \sin (k \phi)\right]
$$

where $r_{e}$ is the radius as a function of the angle $\phi, a_{0}$ is the zero-order Fourier coefficient, $a_{k}$ and $b_{k}$ are the higher order Fourier coefficients, and $N_{f}$ is the highest frequency term in the expansion. In physical terms, $a_{0}$ is the average radius of the hole, and $\left(a_{1} / 2, b_{1} / 2\right)$ is the 
position vector of the hole centre with respect to the chosen origin. The Fourier coefficients with $k>1$ account for any deviations from circularity, with $\sqrt{{a_{k}{ }^{2}+b_{k}{ }^{2}}^{2}}$ measuring the amplitude of the deviation component having $k$ lobes. $N_{f}$ should therefore be chosen to be at least as high as the maximum expected number of lobes around the hole. The case of the three-lobed circle considered in [3], for example, requires $N_{f} \geq 3$.

The contour can be estimated by minimising the objective function

$$
F=\int_{R_{1}}\left[I(x, y)-I_{1}\right]^{2} \mathrm{~d} A+\int_{R_{2}}\left[I(x, y)-I_{2}\right]^{2} \mathrm{~d} A
$$

where $R_{1}$ is the region inside the contour and $R_{2}$ is the region outside the contour, both with respect to the contour $r_{e}(\phi)$, and $\mathrm{d} A$ represents an element of area. The intensities $I_{1}$ and $I_{2}$ can be calculated directly, whereas calculation of the coefficients $a_{0}, \ldots a_{N_{f}}$ and $b_{1}, \ldots b_{N_{f}}$ is a nonlinear problem and requires an iterative technique, implemented here using the GaussNewton method. Appendix 1 gives the details of how $I_{1}, I_{2}$ and the coefficients $a_{0}, \ldots a_{N_{f}}$ and $b_{1}, \ldots b_{N_{f}}$ are calculated. This edge detection method is referred to as the Fourier descriptor (FD) method in the following section of this paper.

In addition to providing the sub-pixel edge points, the FD method can be used to calculate the normal (or tangential) direction of each sub-pixel point as required by the DMA error compensation method described in section 3. The lowest order approximation consists of a circle of radius $a_{0}$, the normal vector to which is $\mathbf{n}_{c}=(\cos \phi, \sin \phi)^{\mathrm{T}}$, where T denotes the transpose operator. The higher order terms cause an angular deviation $\beta(\phi)$ between the full expression for the edge contour given by equation (1) and that for the circle, where $\beta$ can be written 


$$
\beta(\phi)=\frac{1}{r_{e}} \frac{\mathrm{d} r_{e}(\phi)}{\mathrm{d} \phi}=\frac{1}{r_{e}} \sum_{k=1}^{N_{f}}\left[-a_{k} k \sin (k \phi)+b_{k} k \cos (k \phi)\right]
$$

The normal to the edge, $\mathbf{n}_{e}$, is therefore $\mathbf{n}_{e}=\mathbf{M} \mathbf{n}_{c}$, where $\mathbf{M}$ is the rotation matrix

$$
\mathbf{M}=\left(\begin{array}{cc}
\cos (\beta) & -\sin (\beta) \\
\sin (\beta) & \cos (\beta)
\end{array}\right)
$$

\subsection{Validation on simulated and experimental data}

In this sub-section, the robustness of the FD method was verified with both simulated and experimental data. The simulated data were prepared with dark circles of 20, 10 and 5 pixels radii, which have a similar footprint on the image plane to the $8 \mathrm{~mm}, 4 \mathrm{~mm}$ and $2 \mathrm{~mm}$ radius holes, respectively, in the experimental test object. The circles were then smoothed with a square convolution kernel of side $N_{k}=5$ pixels in order to simulate the optical blur similar to that observed in the fringe projection camera. Gaussian random noise was subsequently added to the smoothed simulated texture images, with a standard deviation equal to $I_{w} / S$, where $I_{w}$ is the mean intensity of the white (lighter) regions of the image and $S$ is the signal to noise ratio (SNR). In the simulations, the SNR level was kept at 100; $I_{1}$ and $I_{2}$ took the values 0 and 64, respectively. The errors in the estimated centres and radii using the FD method were calculated from 100 independent simulations.

The results obtained are shown in Table 1 in the format $\delta \bar{r} \pm \sigma_{r}$ for radii and $\left(\delta \bar{x} \pm \sigma_{x}, \delta \bar{y} \pm \sigma_{y}\right)$ for centres, where $\delta \bar{r}, \delta \bar{x}$ and $\delta \bar{y}$ are the mean shifts in the computed radii and centre coordinates, and $\sigma_{r}, \sigma_{x}$ and $\sigma_{y}$ are the corresponding standard deviations. All the values are expressed in units of one thousandth of a pixel. The larger circles have smaller errors than those of the smaller circles, and the FD method is seen to be capable of estimating the centre accurate to one hundredth of a pixel in the presence of an SNR of 100. 
The results obtained on experimental data from the test object using the FD method were compared with those obtained using the sub-pixel detection method [14]. Significant differences in the edge location were apparent. Figure 8 is an example where the FD results (red lines) differ in radius by up to a pixel from the sub-pixel detection method (green dots) [14]. For larger holes which have more than 20 pixels along their diameter, both methods provide similar results. For smaller holes (less than 20 pixels along the diameter), the subpixel method based on partial area effect [14] provides an estimate of the edge that is distorted into the shape of a rectangle with rounded corners, whereas the FD method provides an undistorted circular result.

\section{DMA error compensated experimental results}

The algorithm described in Sec. 3.2 was employed to compensate for the DMA error on and around the holes detected from the image processing step. The value of $R_{P}$, estimated using the procedure described in [13], was 5.0 pixels. Figure 10 shows an example $Z$ distribution around a $4 \mathrm{~mm}$ radius hole before and after the DMA error compensation. The unexpected distortion around the edge is well suppressed, thus enabling more accurate radius estimation.

The radius and the centre of each hole were calculated as before by fitting a 3D circle to the $X$, $Y$, and $Z$ data on the hole edge points returned by the FD method. As the edge points are localised with sub-pixel resolution, the coordinate data along the edges were also obtained to sub-pixel resolution from the $X, Y$ and $Z$ arrays by interpolation.

The radii and centre estimates for all the holes in the plate were then compared with the corresponding 'ground truth' measurements obtained by the CMM. The differences between the optical and CMM measurements of radius were grouped according to nominal hole radius; within each group the mean and root mean square (RMS) of the differences were calculated. 
The RMS distances between the optical and CMM estimates of circle centres were also calculated. As the hole centres estimated by the two measurement systems are in different coordinate systems, they were aligned first using a singular value decomposition (SVD) method [15] before the comparison.

Table 2 provides the mean and RMS error in the estimated radius for the five different hole sizes, using the original uncompensated coordinate data. The RMS error in distance between the estimated centres of the holes and the true centres is also shown. Table 3 shows the corresponding results with the DMA error compensation method as outlined above.

The results given in Tables 2 and 3 are compared graphically in Fig. 11. It is clear that the use of compensated coordinate data results in significant improvements in the estimated radius, compared to the original uncompensated data. For the uncompensated results on the larger holes, RMS values are similar to the mean error, indicating that the RMS errors are dominated by the systematic rather than random errors. Both are in the 200-300 $\mu \mathrm{m}$ level for 15, 8 and $4 \mathrm{~mm}$ radius holes. After compensation, the mean error is reduced to $25 \mu \mathrm{m}$ or less, i.e. over an order of magnitude reduction. The RMS error is reduced to $50 \mu \mathrm{m}$ or less, i.e. a factor of 5 or so improvement. The accuracy of the estimation of hole centre location, on the other hand, improves only a little after DMA error compensation, with the largest improvement for the largest holes $(70 \mu \mathrm{m}$ instead of $100 \mu \mathrm{m})$ and in fact is marginally worse after correction for the smallest holes (radius $1 \mathrm{~mm}$ ). The reason for this can be seen from Figure 4(b), where the anti-symmetrical nature of the height measurement error causes - in this idealised case - no shift in the centre location. 


\section{Discussion}

As seen in the previous section, the proposed DMA error compensation method combined with the Fourier descriptor based closed contour detection method can successfully bring down the error in estimated radius by up to 4-11 times for holes of radius larger than $2 \mathrm{~mm}$. For the $1 \mathrm{~mm}$ radius holes, the improvement in estimated radius was however significantly worse. In this section a possible explanation for this observation is proposed.

The number of pixels along the diameter of a $1 \mathrm{~mm}$ radius hole is less than 10 in the captured images. This means that the effective radius of the PSF, at 5 pixels, is comparable to the radius of the hole. The model proposed in section 3.1, on the other hand, assumes a straight edge to the hole over the length scale of the PSF, and hence implicitly assumes the PSF radius to be small compared to the hole radius. This can be seen in Fig. 12, where for simplicity the point of interest $\left(x_{c}, y_{c}\right)$ lies on the $x$ axis, i.e. $y_{c}=0$. The camera PSF is seen to include the two cross-hatched regions that in the model lie within the hole, and are therefore ignored, but in reality contribute scattered light to the detected signal. As these two regions lie to the left of the centre point of the integration circle, the $\omega_{x}$ values of the scattered light from them balances those from the light scattered by corresponding regions to the right of the integration circle centre point. The original uncompensated measurement will therefore be less biased than is assumed in the model. The model will as a result tend to over compensate for the error and thus give a smaller reduction in error than for the larger holes. An improved model that takes account of the curvature of the hole edge within the PSF can be expected to result in a smaller error, however such a refinement is beyond the scope of the current paper.

It should also be noted that the scanner used in this study had a relatively large field of view of about $1 \times 1 \mathrm{~m}^{2}$ at a working distance of $2.5 \mathrm{~m}$. Characterising the geometry of holes with 
radius less than $2 \mathrm{~mm}$ could be achieved more accurately with a system which has a narrower field of view and a smaller point spread function.

\section{Conclusions}

Automated assembly often requires the ability to accurately estimate the location and geometry of holes. Fringe-projection-based 3D optical scanners would seem ideal for this purpose, being based on a non-contact measurement technique, especially on large freeform samples. However, evidence was found in this study that problems will occur wherever there are reflectivity or depth discontinuities, hindering the accurate estimation of hole sizes. In this paper, a solution to compensate for the errors arising due to these discontinuities around holes is presented, and an effective sub-pixel closed edge detection method based on a Fourier descriptor is proposed to facilitate the error compensation scheme. The FD method was found to be successful at detecting small holes which only have a few pixels along the edge. With the presented error compensation method, the discontinuity-induced systematic errors around the edges were well suppressed, and the subsequently estimated hole radii were much closer to the true values. The estimated RMS error in radius was decreased by a factor of $4-11 \times$ for the holes larger than a radius of $2 \mathrm{~mm}$. The estimated centre positions were also on average slightly improved.

\section{Acknowledgements}

The authors are grateful to Mr Ben Horton for the design of the test panel, and acknowledge financial support from the Engineering and Physical Sciences Research Council under the Light Controlled Factory project EP/K018124/1. This work was also supported by National Nature Science Foundation of China (Grant No. 61421002), and the Fundamental Research Funds for the Central Universities 


\section{References}

1. C. Munkelt, P. Kühmstedt, L. Aschermann, F. Seidel, ‘Automatic complete high-precision optical 3D measurement of air cooling-holes of gas turbine vanes for repair', Proc. of SPIE 9525, 9525121-95251217, 2015.

2. G. Y. Tian, Z. X. Zhao, R. W. Baines, P. Corcoran, 'A miniaturised sensor for deep hole diameter measurement', Precision Engineering, 23, 236-242 (1999).

3. Flack, D. Measurement Good Practice Guide No.41 - CMM measurement strategies. Teddington : National Physical Laboratory, 2001 updated 2014.

4. S. S. Gorthi and P. Rastogi, 'Fringe Projection Techniques: Whither we are?’, Optics and Lasers in Engineering, 48(2), 133-140 (2010).

5. S. Zhang, 'Recent progresses on real-time 3D shape measurement using digital fringe projection techniques’, Optics and Lasers in Engineering, 48,149-158 (2010).

6. X. Y. Su, Q. C. Zhang, 'Dynamic 3-D shape measurement method: a review’, Optics and Lasers in Engineering, 48(2), 191-204 (2010).

7. P. Brakhage, M. Heinze, G. Notni, and R. Kowarschik, 'Influence of the pixel size of the camera on 3d-measurementswith fringe projection,' in Optical Measurement Systems for Industrial Inspection III, W. Osten, K. Creath, and M. Kujawinska, eds., Proceedings of SPIE 5144, 478-483 (2003).

8. H. Yue, H. G. Dantanarayana, Y. Wu and J. M. Huntley, 'Reduction of systematic errors in structured light metrology at discontinuities in surface reflectivity', Optics and Lasers in Engineering (in preparation, to be submitted January 2018).

9. Y. Wu, H. Dantanarayana, H. Yue, J. M. Huntley, 'Accurate characterisation of hole geometries by fringe projection profilometry', in Videometrics, Range Imaging, and 
Applications XIV, F. Remondino and M. R. Shortis, eds., Proceedings of SPIE 10332, 1033204 (2017).

10. H. O. Saldner and J. M. Huntley, 'Temporal phase unwrapping: application to surface profiling of discontinuous objects’, Applied Optics, 36(13), 2770-2775 (1997).

11. J. M. Huntley and H. O. Saldner, 'Error-reduction methods for shape measurement by temporal phase unwrapping', J. Opt. Soc. Am. A, 14(12), 3188-3196 (1997).

12. J. Canny, ‘A computational approach to edge detection', IEEE Transactions on Pattern Analysis and Machine Intelligence, 8(6), 679-698 (1986).

13. F. P. Wang, 'The measurement of the point spread function in optical system with CCD', JGTC Int. Journal of Gannan Teachers College, Papers (06), 17-18 (2005).

14. T. P. Agustín, K. Karl, A. F. Miguel, S. C. Daniel. 'Accurate subpixel edge location based on partial area effect', Image and Vision Computing, 31, 72-90 (2013).

15. M. Sjödahl and B. F. Oreb, 'Stitching interferometric measurement data for inspection of large optical components’, Optical Engineering, 41(2), 403-408 (2002).

16. J. J. McKeown, D. Meegan and D. Sprevak, 'An introduction to unconstrained optimisation’, IOP Publishing, Bristol (1990). 


\section{Appendix 1}

This appendix describes some of the mathematical details of the Fourier Descriptor method first introduced in section 4.

Minimisation of Equation (6) with respect to the two intensity values $I_{1}$ and $I_{2}$ leads to the following pair of equations:

$$
\begin{aligned}
& \frac{\partial F}{\partial I_{1}}=-2 \int_{R_{1}}\left[I(x, y)-I_{1}\right] \mathrm{d} A \\
& \frac{\partial F}{\partial I_{2}}=-2 \int_{R_{2}}\left[I(x, y)-I_{2}\right] \mathrm{d} A
\end{aligned}
$$

The derivatives with respect to the Fourier coefficients $a_{0}, \ldots a_{N_{f}}$ and $b_{1}, \ldots b_{N_{f}}$ reduce to line integrals along the contour $C$ :

$$
\begin{aligned}
& \frac{\partial F}{\partial a_{0}}=2\left(I_{2}-I_{1}\right) \int_{C}(I-\bar{I}) r_{e} \mathrm{~d} \phi \\
& \frac{\partial F}{\partial a_{k}}=2\left(I_{2}-I_{1}\right) \int_{C}(I-\bar{I}) r_{e} \cos (k \phi) \mathrm{d} \phi, \quad k=1,2, \ldots, N_{f} \\
& \frac{\partial F}{\partial b_{k}}=2\left(I_{2}-I_{1}\right) \int_{C}(I-\bar{I}) r_{e} \sin (k \phi) \mathrm{d} \phi, \quad k=1,2, \ldots, N_{f}
\end{aligned}
$$

where

$$
\bar{I}=\left(I_{1}+I_{2}\right) / 2
$$

For a given current estimate of the contour, equation (9) is easily solved to give the least squares estimator for $I_{1}$ and $I_{2}$ as follows:

$$
I_{i}=\frac{\int_{R_{i}} I(x, y) \mathrm{d} A}{\int_{R_{i}} \mathrm{~d} A}, \quad i=1,2 .
$$


Equation (10) is, however, nonlinear and should be solved by an iterative technique (GaussNewton method in this study). The iterative scheme used involves solving for the vector $\mathbf{d}$

$$
\mathbf{H d}=-\mathbf{g}
$$

where $\mathbf{g}$ donates the gradient vector of $F$ (see equation (10)) with respect to each of the free parameters, and $\mathbf{H}$ is the Hessian matrix (matrix of second derivatives such that $\left.H_{i j}=\partial^{2} F / \partial x_{i} \partial x_{j}\right)[16]$. The solution vector $\mathbf{x}$ is then updated as follows at the $k$ th step:

$$
\mathbf{x}^{(k+1)}=\mathbf{x}^{(k)}+\mathbf{d}
$$

Equations (13) and (14) are iterated from an initial estimate for $\mathbf{x}$ until convergence is achieved. The format for $\mathrm{x}$ is:

$$
\mathbf{x}=\left(\begin{array}{c}
a_{0} \\
a_{1} \\
a_{2} \\
\cdot \\
a_{N f} \\
b_{1} \\
b_{2} \\
\cdot \\
b_{N f}
\end{array}\right)
$$

The entries for the Hessian matrix can be calculated as follows:

$$
\begin{aligned}
& \frac{\partial^{2} F}{\partial a_{j} \partial a_{k}}=2\left(I_{2}-I_{1}\right) \int_{C}\left(r \frac{\partial I}{\partial r_{e}}+(I-\bar{I})\right) \cos (j \phi) \cos (k \phi) \mathrm{d} \phi \\
& \frac{\partial^{2} F}{\partial b_{j} \partial b_{k}}=2\left(I_{2}-I_{1}\right) \int_{C}\left(r \frac{\partial I}{\partial r_{e}}+(I-\bar{I})\right) \sin (j \phi) \sin (k \phi) \mathrm{d} \phi \\
& \frac{\partial^{2} F}{\partial a_{j} \partial b_{k}}=2\left(I_{2}-I_{1}\right) \int\left(r \frac{\partial I}{\partial r_{e}}+(I-\bar{I})\right) \sin (j \phi) \cos (k \phi) \mathrm{d} \phi
\end{aligned}
$$

Once the $\mathbf{x}$ vector has been calculated, sub-pixel edge coordinates can be calculated at any desired $\phi$ value by equation (5). 
When implementing the line integrals along the contour (Equations (10) and (16)), the continuous integrals are replaced by sums over the relevant sampled quantities, where the number of samples should be more than $2 N_{f}$ to ensure adequate sampling according to the Shannon sampling criterion. Computational effort for these two line integrals is significantly reduced, compared to a direct implementation, through the use of a 1-D fast Fourier transform.

In order to increase robustness if the initial parameter estimate vector is insufficiently accurate, a simple modification to equation (14) can be applied [16]:

$$
\mathbf{x}^{(k+1)}=\mathbf{x}^{(k)}+\gamma \mathbf{d}
$$

The scalar $\gamma$ is normally chosen to be 1 , but if $F$ is increased, the value of $\gamma$ which minimizes $F$ is used instead. A further modification involves testing for negative eigenvalues of $\mathbf{H}$. If they are found, a scaled identity matrix is added to $\mathbf{H}$ to ensure all eigenvalues are positive. 


\section{Tables}

Table 1. The error of radii and centre estimation of simulated circles with sizes between 5-20 image pixels. The simulated images were smoothed with a kernel of $N_{k}=5$ and Gaussian noise of SNR = 100 was added. All errors are given in units of one thousandth of a pixel.

\begin{tabular}{|c|c|c|c|}
\hline & Radius=20 pixels & Radius=10 pixels & Radius=5 pixels \\
\hline$\delta \bar{r} \pm \sigma_{r}$ & $-58.8 \pm 58.9$ & $-61.2 \pm 61.4$ & $171.2 \pm 172.3$ \\
\hline$\left(\delta \bar{x} \pm \sigma_{\delta x}, \delta \bar{y} \pm \sigma_{\delta y}\right)$ & $(-0.55 \pm 5.3,-0.36 \pm$ & $(-0.38 \pm 9.7,-0.93 \pm$ & $(-1.9 \pm 11.5,-1.1 \pm$ \\
& $5.4)$ & $9.2)$ & $11.7)$ \\
\hline
\end{tabular}

Table 2. Errors in estimated hole radius and location before DMA error compensation.

\begin{tabular}{|l|c|c|c|c|c|}
\hline $\begin{array}{l}\text { Error type (mm) } \\
\text { hole radius } \\
\text { (mm) }\end{array}$ & 15 & 8 & 4 & 2 & 1 \\
\hline $\begin{array}{l}\text { Estimated radius } \\
\text { mean error }\end{array}$ & 0.2235 & 0.3111 & 0.2900 & 0.3582 & 0.4694 \\
\hline $\begin{array}{l}\text { Estimated radius } \\
\text { RMS error }\end{array}$ & 0.2243 & 0.3130 & 0.2942 & 0.3591 & 0.4709 \\
\hline $\begin{array}{l}\text { Centres } \\
\text { alignment RMS } \\
\text { error }\end{array}$ & 0.0991 & 0.0273 & 0.0257 & 0.0336 & 0.0293 \\
\hline
\end{tabular}


Table 3. Errors in estimated hole radius and location with DMA error compensation.

\begin{tabular}{|l|c|c|c|c|c|}
\hline $\begin{array}{l}\text { Error type (mm) } \\
\text { /hole radius } \\
(\mathrm{mm})\end{array}$ & 15 & 8 & 4 & 2 & 1 \\
\hline $\begin{array}{l}\text { Estimated radius } \\
\text { mean error }\end{array}$ & -0.0074 & -0.0110 & -0.0258 & 0.0825 & 0.3579 \\
\hline $\begin{array}{l}\text { Estimated radius } \\
\text { RMS error }\end{array}$ & 0.0204 & 0.0324 & 0.0512 & 0.0875 & 0.3602 \\
\hline $\begin{array}{l}\text { Centres } \\
\text { alignment error }\end{array}$ & 0.0669 & 0.0113 & 0.0239 & 0.0319 & 0.0413 \\
\hline
\end{tabular}




\section{Figures}

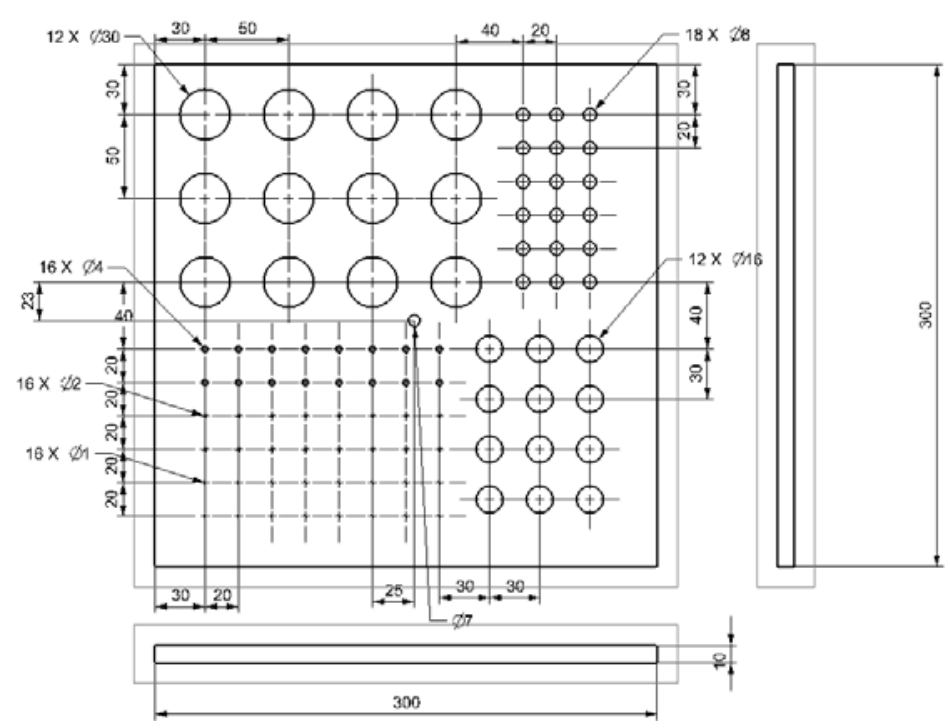

Figure 1. Aluminium test panel with arrays of holes, the diameters of which range from 30 $\mathrm{mm}$ down to $1 \mathrm{~mm}$. 

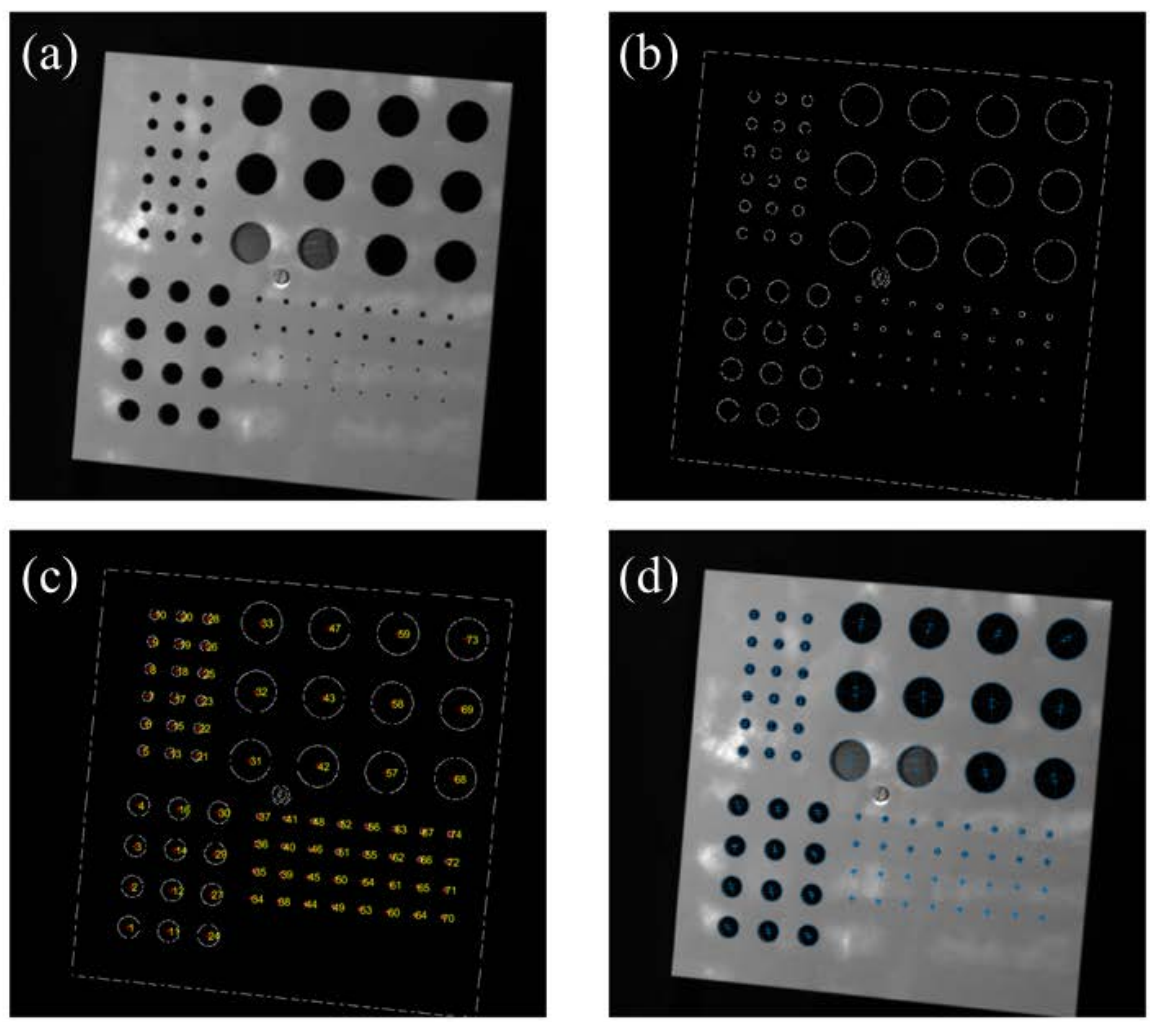

Figure 2. Standard image processing procedures applied to the texture image (a). Edges within the image are detected by a Canny operator (b); connected edge points are given unique labels (c); ellipse fitting to each labelled edge identifies the holes, and determines the hole centres and circumference pixels on the texture image plane (d). 


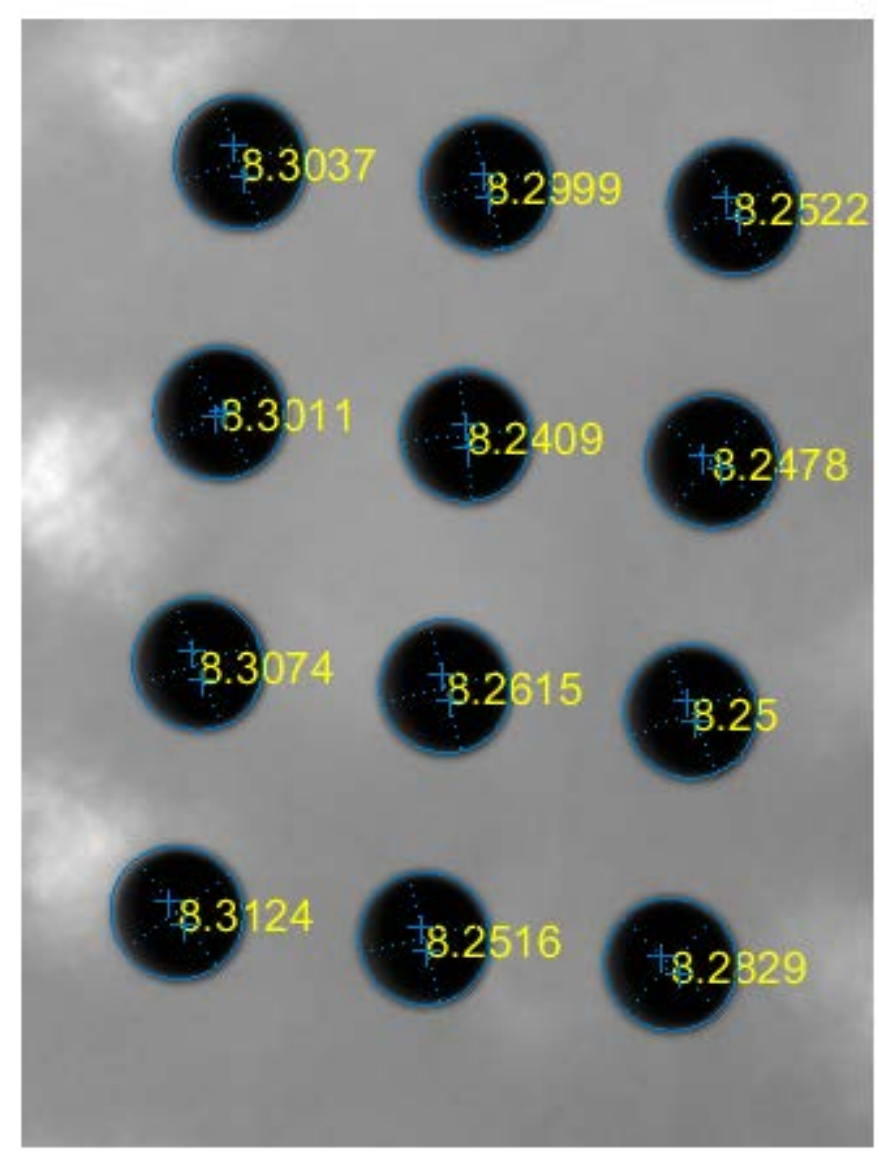

Figure 3. Estimated radius (units: $\mathrm{mm}$ ) for each of the holes with a nominal radius of $8 \mathrm{~mm}$, obtained by fitting an ellipse to the 3D $X, Y, Z$ fringe projection data on the edge pixels of the holes. The holes occupy the bottom left corner of the test plate shown in Fig. 2(a). 
(a)

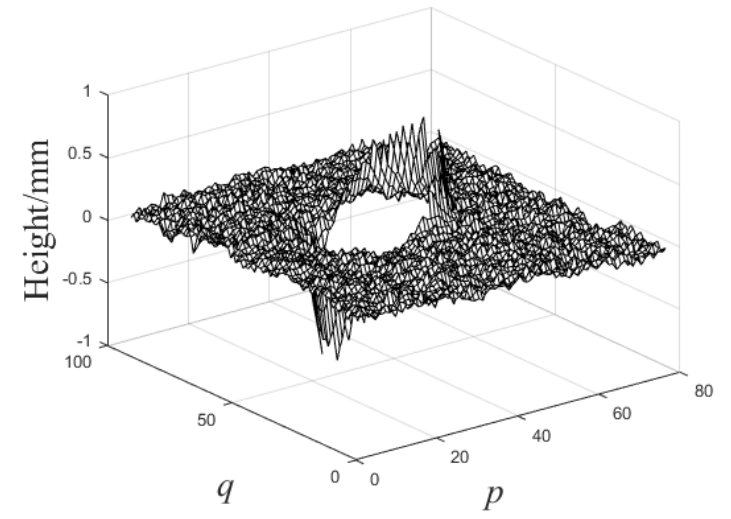

(b)

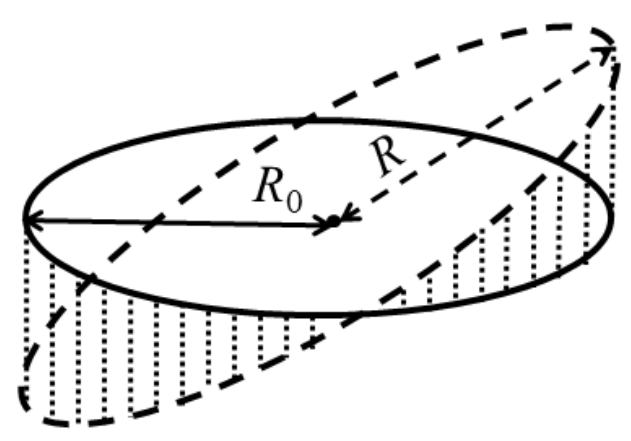

Figure 4. DMA error around the edge of a hole. (a) Experimental height distribution in neighbourhood of a 4-mm radius hole measured by fringe projection, where indices $(p, q)$ denote the location of a given pixel within the sub-image; (b) an illustration of how DMA error affects the estimation of hole radius. 
(a)

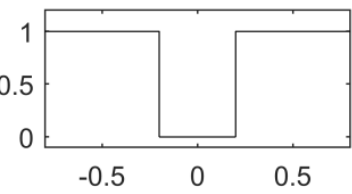

(b)

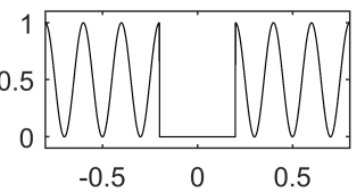

(c)

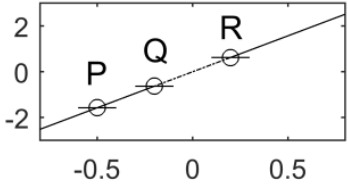

(d)
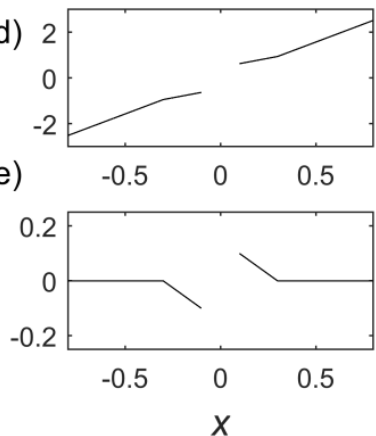

Figure 5. 1-D model of DMA formation. Image-plane intensity distribution $I(x)$ for sample containing hole, with uniform illumination (a) and fringe illumination (b), and corresponding unwrapped phase $\omega_{x}(x)$ (c), assuming 'zero-width' point spread function. Modelled phase $\bar{\omega}_{x}(x)(\mathrm{d})$ and apparent height profile (e) for the case of a PSF diameter equal to $10 \%$ of the field of view and a $45^{\circ}$ projection angle. 


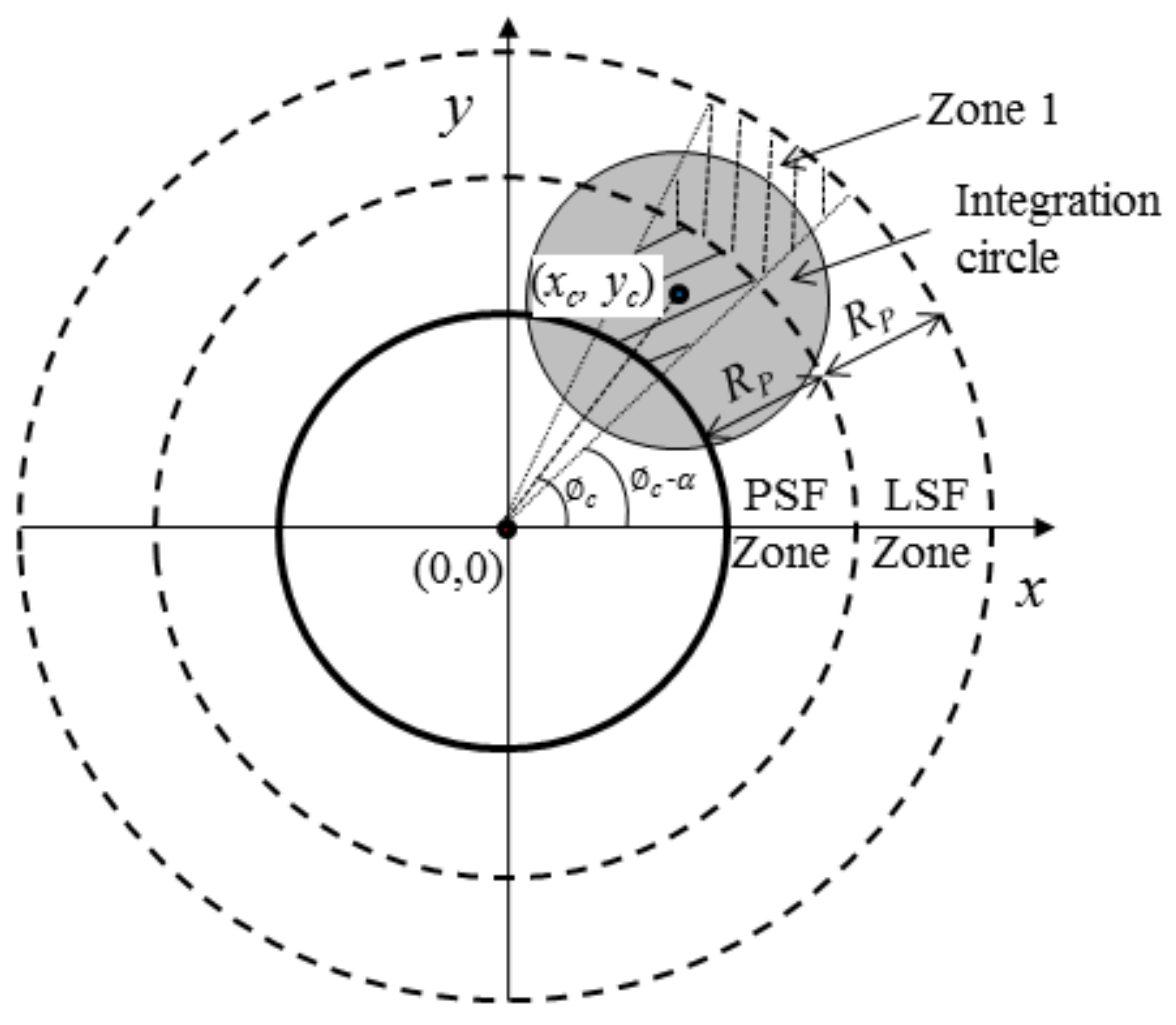

Figure 6. Schematic of Point Spread Function (PSF) and Least Squares Fitting (LSF) zones around a hole (solid line). The grey area denotes the area of integration of the compensation model to correct the phase value at pixel $\left(x_{c}, y_{c}\right)$. 
(a)

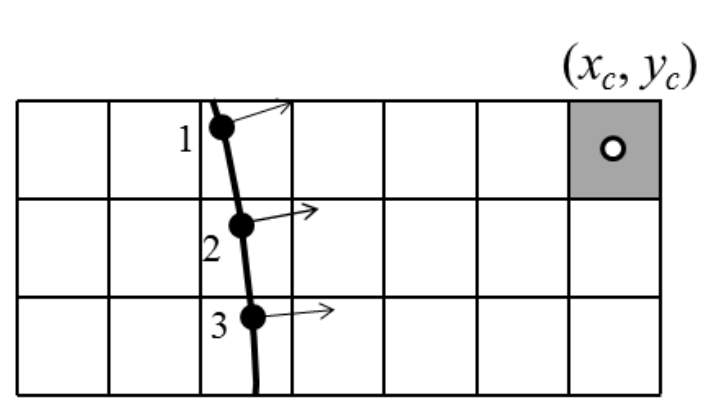

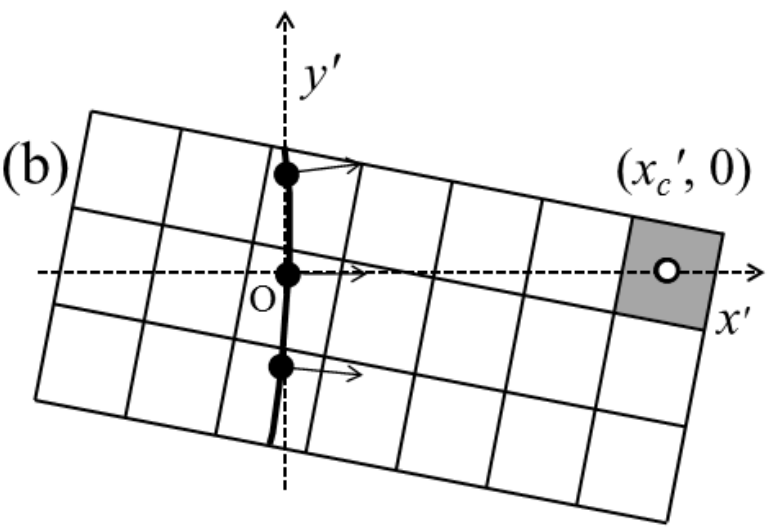

Figure 7(a) Correction of phase at grey-shaded pixel, with centre coordinates $\left(x_{c}, y_{c}\right)$, in the neighbourhood of a hole edge (solid black curve). Labels $(1,2,3)$ denote three sub-pixel points on the edge of the hole, of which point 2 is closest to $\left(x_{c}, y_{c}\right)$. Arrows indicate the direction of the edge normal. (b) Rotation of the pixel grid onto coordinate system $\left(x^{\prime}, y^{\prime}\right)$ which has its origin $\mathrm{O}$ at the closest sub-pixel edge point to $\left(x_{c}, y_{c}\right)$, with $x^{\prime}$ and $y^{\prime}$ respectively perpendicular and parallel to the hole edge. 
(a)

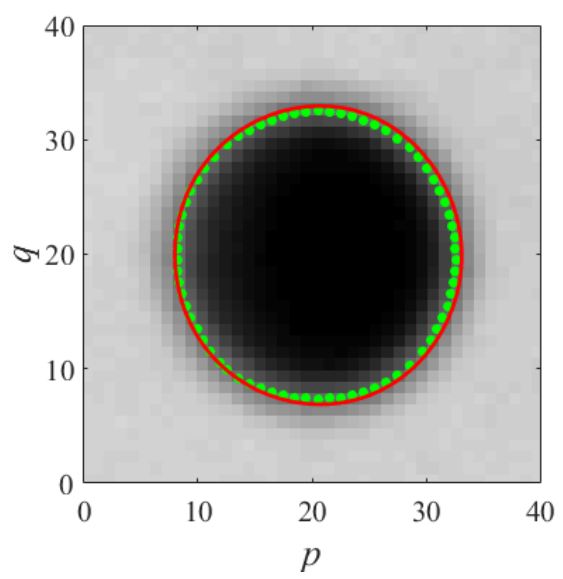

(b)

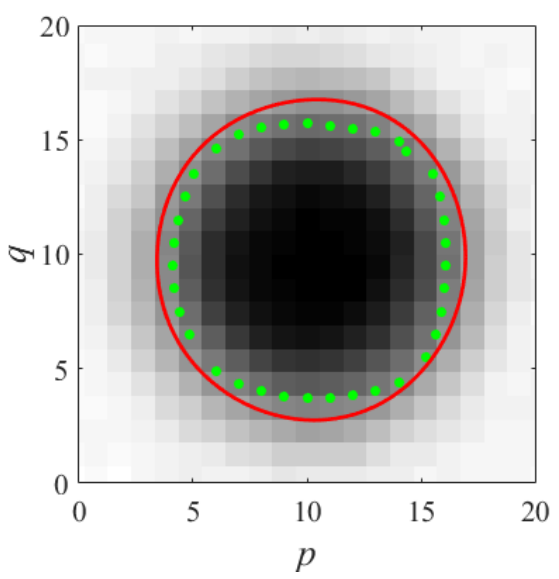

(c)

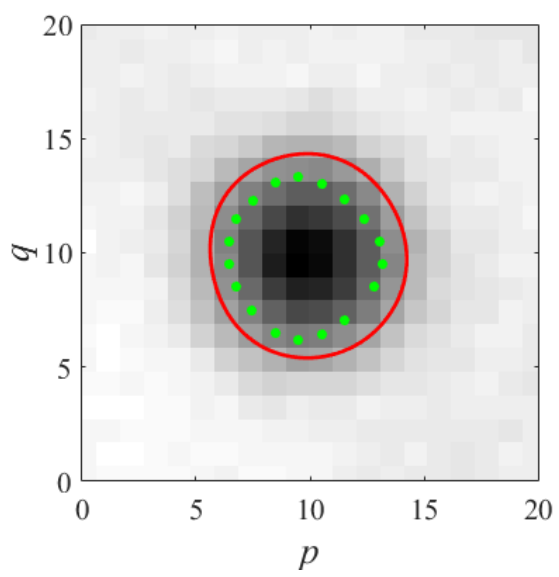

Figure 8. Estimated edge of a hole from one of the sub-images with the sub-pixel edge detection method from [14] (green dots) and the Fourier Descriptor method (continuous red line) for holes of radius $4 \mathrm{~mm}$ (a), $2 \mathrm{~mm}$ (b) and $1 \mathrm{~mm}$ (c). 


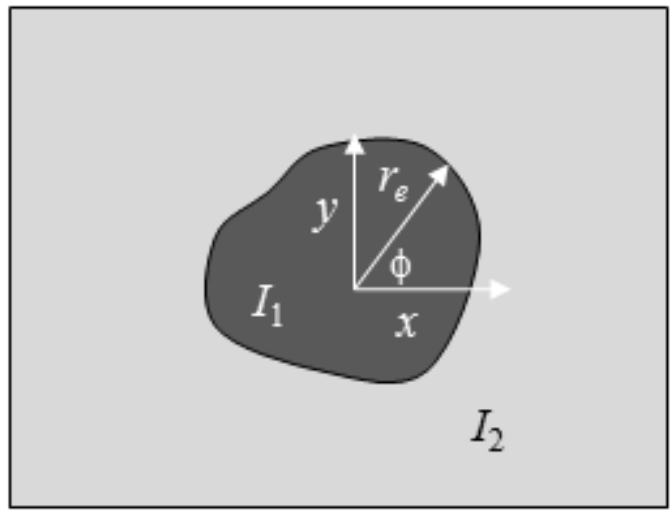

Figure 9. Model for a hole within a texture image $I(x, y)$ as a closed contour of arbitrary shape, separating regions of constant intensity magnitudes $I_{1}$ and $I_{2}$ inside and outside the contour, respectively. 
(a)

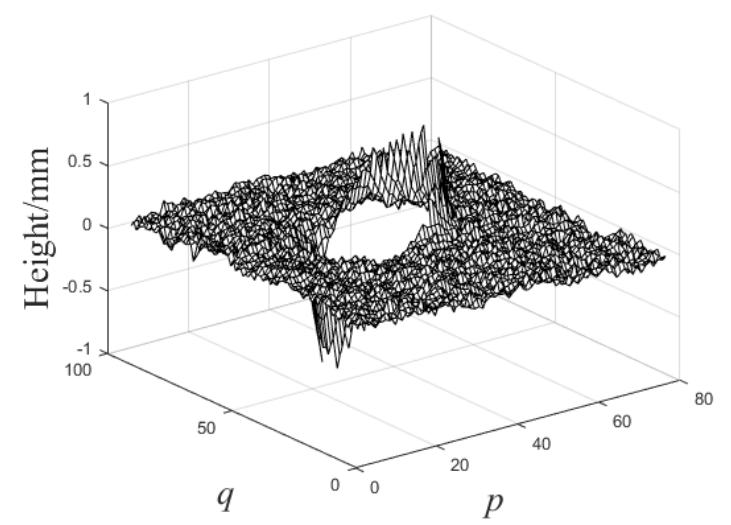

(b)

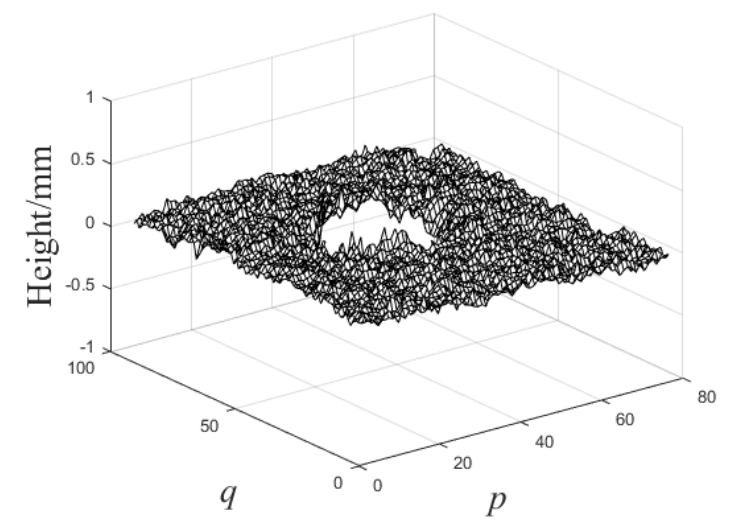

Figure 10. Height distribution around a $4 \mathrm{~mm}$ radius hole (a) before and (b) after DMA error compensation. 


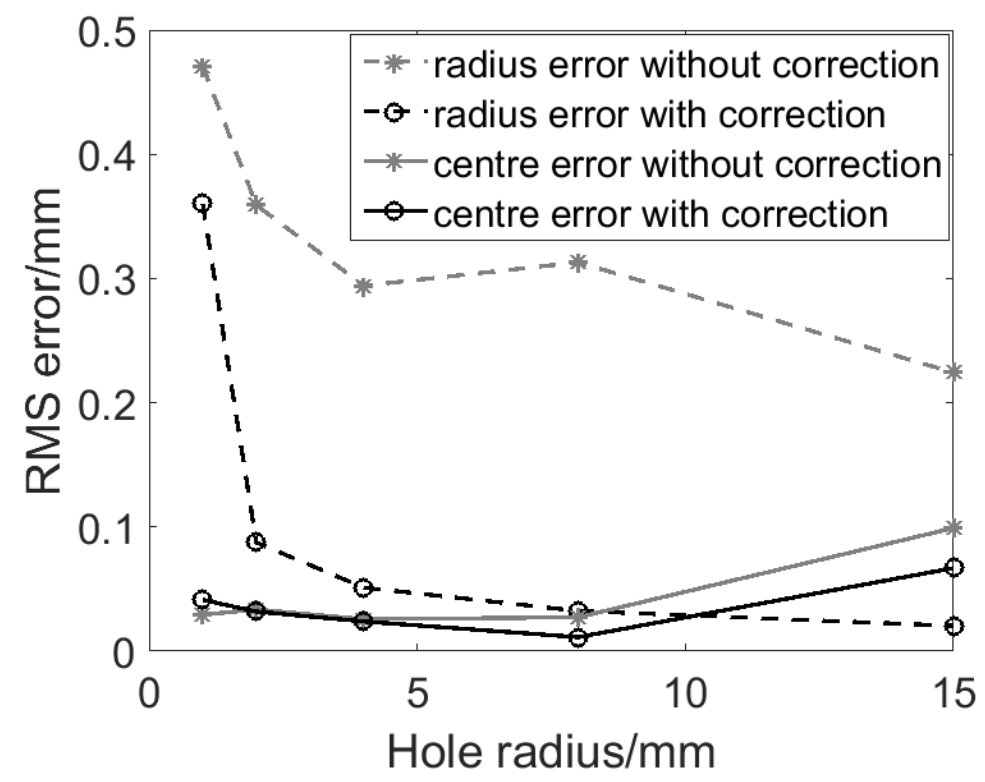

Figure 11. Root mean square radius and centre coordinate errors before and after DMA compensation for hole radii ranging from 1-15 mm. 


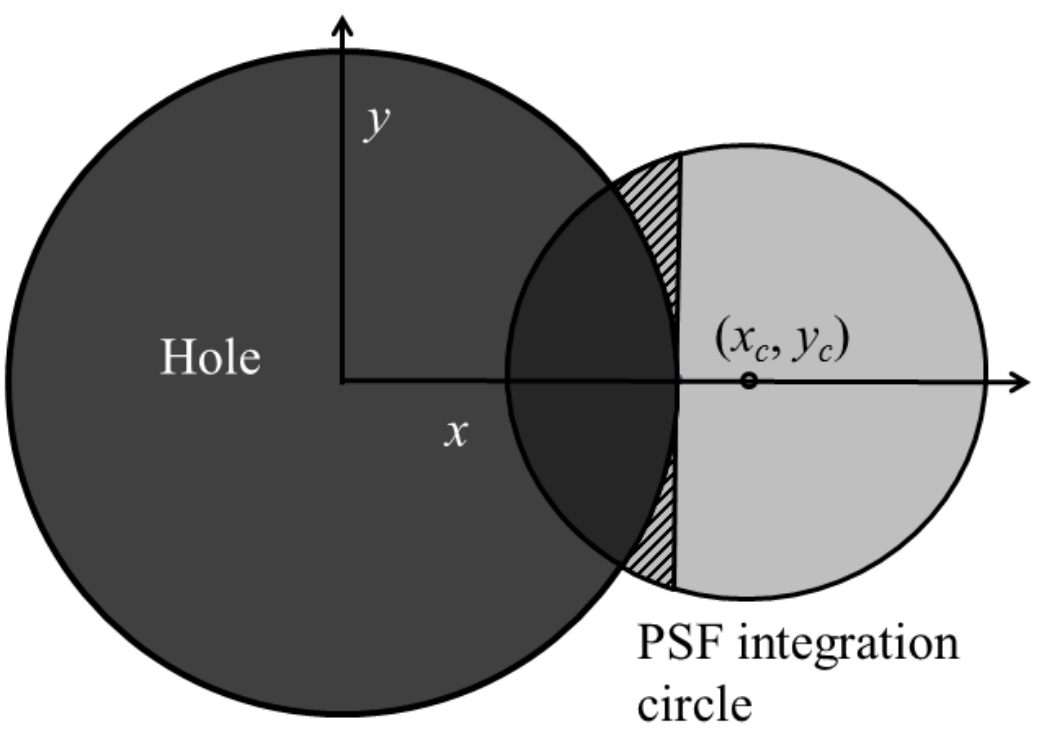

Figure 12. Limitation of DMA model when the PSF radius approaches that of the hole.

Scattered light from the points within the cross-hatched regions contributes to the optical signal measured at point $\left(x_{c}, y_{c}\right)$, but is not accounted for in the model. 